\title{
VISUAL OUTCOME OF PATIENTS WITH IDIOPATHIC ISCHAEMIC AND NON-ISCHAEMIC RETINAL VASCULITIS
}

\author{
H. E. PALMER, M. R. STANFORD, M. D. SANDERS and E. M. GRAHAM \\ London
}

\begin{abstract}
SUMMARY
This study investigated whether patients with ischaemic retinal vasculitis have a worse visual outcome than those with non-ischaemic disease. A retrospective study was made of 53 patients with idiopathic retinal vasculitis (RV), with minimum 5 year follow-up. Patients were categorised into ischaemic and non-ischaemic groups by fluorescein angiography. Visual outcome was determined by visual acuity at their last attendance. Twenty patients (38 eyes) had ischaemic $R V$; 33 patients (63 eyes) had non-ischaemic $R V$. At presentation there was no significant difference between the groups in the proportion of eyes with poor vision (6/60 or less). Ischaemic $R V$ patients had a worse visual outcome than those with non-ischaemic RV: 13 of $38(34 \%)$ eyes in the ischaemic group had a final poor vision compared with 4 of $63(6 \%)$ eyes in the non-ischaemic group (Fisher's exact test, $p=0.0005$ ).
\end{abstract}

Retinal vasculitis (RV) is an inflammatory disease of the retina, uveal tract and vitreous, which predominantly affects young people and can lead to blindness. It is a disease which is characterised by intraocular inflammation with involvement of the retinal blood vessels. When the inflammation is predominantly at the pars plana, the disease is termed intermediate uveitis. RV can occur in isolation (idiopathic RV), or in association with systemic inflammatory conditions such as sarcoidosis and Behçet's disease, infections or neoplasia. ${ }^{1}$ The two main causes of visual loss in $\mathrm{RV}$ are cystoid macular oedema and vitreous haemorrhage, from new vessel formation consequent upon retinal ischaemia, which can occasionally lead to traction retinal detachment. ${ }^{2}$

\footnotetext{
From: Medical Eye Unit, St Thomas' Hospital, London, UK. Correspondence to: Miss H. E. Palmer, Department of Ocular Immunology, Rayne Institute, St Thomas' Hospital, London SE1 7EH, UK.
}

Idiopathic RV can be classified into ischaemic and non-ischaemic forms; idiopathic ischaemic RV is like 'Eales disease', named after Henry Eales who first reported the disease in $1880 .^{3}$ To date, there is no evidence that patients with ischaemic RV have a worse visual prognosis than those with non-ischaemic disease. Indeed, ischaemic disease has been reported by Elliot ${ }^{4}$ to be associated with a good prognosis. In a prospective study between 1970 and 1991, Atmaca et al. ${ }^{5}$ also found that the visual outcome for Eales patients was favourable. Other papers on Eales disease, whilst giving detailed descriptions of the disease manifestations, have not focused upon visual outcome. ${ }^{6,7}$ Patients with pars planitis have also been found to have a good visual outcome. ${ }^{8}$ It was our clinical impression that patients with ischaemic RV fared less well. The main aim of this study was, therefore, to determine whether patients with ischaemic RV have a worse visual outcome than those with non-ischaemic disease and to assess the causes of visual failure in each group.

\section{METHODS}

A retrospective study was undertaken of $\mathrm{RV}$ patients who had been attending the Medical Eye Unit at St Thomas' Hospital for a minimum follow-up period of 5 years, for whom there was accurate documentation of ophthalmic features at least every 6 months. The age at presentation and sex of each patient were recorded, as were details of their smoking habits and duration of RV before referral. At presentation, all the patients had had a full ophthalmological and general medical history taken, and a thorough examination which included Snellen and reading acuities, colour vision testing, slit lamp and fundal examination and grading of intraocular activity. Particular note was made of the cause of poor vision (6/60 or less) in an affected eye. The eyes were categorised as having ischaemic or non-ischaemic 
disease on the basis of fluorescein angiography: ischaemic RV was identified by any angiographic evidence of capillary non-perfusion. In addition, the fluorescein angiograms previously performed on all patients were retrospectively reviewed for the presence of macular ischaemia which may not have been identified at the time of the angiogram. Macular ischaemia was defined as closure of perifoveal capillaries identified by an enlarged and/or irregular foveal avascular zone. ${ }^{9}$

Disease course was documented in terms of the number of vitreous haemorrhages and relapse rate. Relapse was defined as an increase in vitreous cellularity, which was usually associated with signs of retinal inflammation such as cystoid macular oedema. ${ }^{10}$ Details of systemic treatment and photocoagulation were noted.

At the patient's last visit Snellen visual acuity was recorded and, again, any cause of visual loss was recorded. As in our previous papers, outcome was graded as follows: grade I ('excellent'), $6 / 6$ or better; grade II ('good'), 6/9 or 6/12; grade III ('fair'), 6/18-6/36; and grade IV ('poor'), 6/60 or worse. A successful visual outcome was defined as either (a) maintenance of visual acuity of $6 / 12$ or better, or (b) improvement by one or more grades, by the last clinic attendance. Patients who did not fulfil either of these criteria were defined as having a poor visual outcome. $^{1 \mathbf{0}, 11}$

\section{RESULTS}

\section{Patients and Characteristics at Presentation}

The patients and their characteristics at presentation are outlined in Table I. The case files of 53 patients with idiopathic RV were studied: 20 had ischaemic RV and 33 had non-ischaemic RV. Forty-eight of these patients had bilateral disease and in 5 patients the disease was unilateral; there was thus a total of 101 affected eyes.

There were no significant differences in either age (Mann-Whitney $U$-test, $p=0.46$ ) or sex distribution (Fisher's exact text, $p=0.22$ ) between the two groups. A greater proportion of the patients with ischaemic RV were smokers compared with their non-ischaemic counterparts: 14 of the $20(70 \%)$ patients with ischaemic RV smoked compared with 18 of $33(54.5 \%)$ with non-ischaemic RV.

One patient with ischaemic RV had orally controlled diabetes mellitus but no diabetic retinopathy. There were no patients with hypertension or a haemoglobinopathy.

\section{Ophthalmic Findings at Presentation}

Disease Duration Before Referral

The majority of patients were initially under the care of their local ophthalmic unit and then referred to St Thomas' Medical Eye Unit for further management. The mean duration of RV prior to referral was 35 months for ischaemic and 32 months for nonischaemic $\mathrm{RV}$ patients.

\section{Visual Acuity and Causes of Grade IV Vision}

The distribution of visual acuity grades at presentation is as shown in Fig. 1. At presentation, overall 16 eyes of 15 patients had grade IV visual acuity, the causes of which are listed in Table II. In over one-third of these eyes (6/16) this was due to cystoid macular oedema (CMO). Vitreous haemorrhage was present in 3 of 16 eyes at presentation, of which 2 occurred in ischaemic RV patients. Although a greater proportion of the eyes with ischaemic $\mathrm{RV}$ had grade IV acuities at this stage (9 of $38(23.6 \%)$ with ischaemic RV compared with 7 of $63(11.1 \%)$ with non-ischaemic RV), this difference was not statistically significant (Fisher's exact test, $p=0.08$ ).

\section{Disease Course}

Follow-up ranged from 5 to 20.2 years and was not significantly different between the two groups (median of 6.2 years for ischaemic and 5.9 for non-ischaemic patients: Mann-Whitney $U$-test, $p=0.87)$.

\section{Disease Relapses}

The number of relapses over the follow-up period for the ischaemic RV patients ranged from nil to 14 (mean of 2.26) and for the non-ischaemic RV patients ranged from nil to 8 (mean of 2.71). When the follow-up period for each patient was taken into

Table I. Patient characteristics at presentation

\begin{tabular}{lccc}
\hline & Ischaemic RV & Non-ischaemic RV & Total RV \\
\hline No. of patients & 20 & 33 & 53 \\
No. of affected eyes & 33 & 68 & 101 \\
Age (years) & $6-50$ & $11-56$ & $6-56$ \\
$\quad$ Range & 25 & 26 & 26 \\
$\quad$ Median & 9 & 10 & 19 \\
Sex & 11 & 23 & 23 \\
$\quad$ Male & & $18(54.5 \%)$ & 32 \\
$\quad$ Female & $14(70 \%)$ & $15(45.5 \%)$ & 21 \\
$\quad$ Smokers & $6(30 \%)$ & & \\
$\quad$ No & & & \\
\hline
\end{tabular}



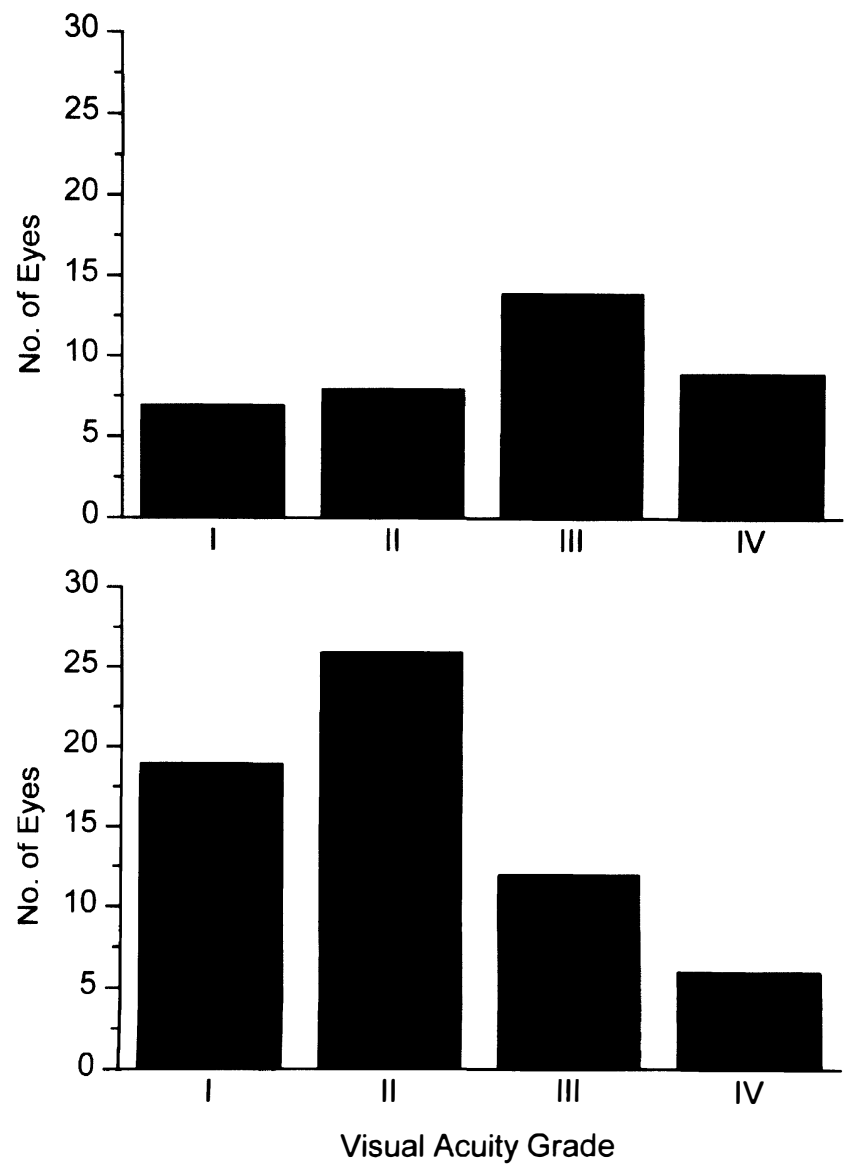

Fig. 1. The distribution of visual acuity grades at the beginning of the study of eyes of ischaemic $R V$ (above) and non-ischaemic $R V$ patients (below). Grade $I, 6 / 6$ or better; grade II, 6/9 or 6/12; grade III, 6/18-6/36; grade IV, 6/60 or worse.

account the median relapse rate was 0.29 per year for the ischaemic $\mathrm{RV}$ patients and 0.36 per year for those with non-ischaemic RV. The difference in these relapse rates between the two groups was not statistically significant (Mann-Whitney $U$-test, $p=0.33$.

\section{Vitreous Haemorrhage}

A significantly higher number of ischaemic RV patients had at least one episode of vitreous haemorrhage compared with the non-ischaemic RV patients ( 9 and 1 respectively; Fisher's exact test, $p=0.0003)$. In the case of the non-ischaemic RV patients this vitreous haemorrhage occurred in one eye and was secondary to neovascularisation due to inflammation rather than ischaemia. The vitreous haemorrhage resolved and the new vessels regressed on systemic treatment leading to a successful visual outcome. Six of the 9 ischaemic RV patients $(75 \%)$ were smokers.

\section{Macular Ischaemia}

On review of all the angiograms performed, 4 patients $(7.5 \%)$ had macular ischaemia. The visual acuities in the affected eyes ranged from $6 / 12$ to $6 / 36$, and resulted in a poor visual outcome in 3 of these eyes. Two of these patients ( 3 eyes) had been documented as having non-ischaemic RV; the remaining 2 patients had ischaemic RV. None of the affected eyes had grade IV vision at the end of the study although 3 eyes fulfilled the criteria for a poor visual outcome.

\section{Treatment}

\section{Photocoagulation}

None of the non-ischaemic RV patients had laser photocoagulation for retinal neovascularisation, although 3 of them had either argon laser or cryotherapy for treatment of supero-temporal retinal holes. Conversely, 5 of the ischaemic RV patients (all of whom had previously had vitreous haemorrhages) had undergone argon or xenon arc laser photocoagulation to treat neovascularisation.

Table II. Causes of grade IV vision (6/60 or less) in RV patients at beginning of study period

\begin{tabular}{llll}
\hline & Patient & Visual acuity & Cause of poor vision \\
\hline Non-ischaemic & WD & $6 / 60$ & Cystoid macular oedema + vitritis \\
& AI & HM & Cystoid macular oedema \\
& HM & OF & Optic atrophy \\
DP & $6 / 60$ & Cataract \\
SP & CF & Macular epiretinal membrane \\
AC & HM & Vitreous haemorrhage \\
JT & $6 / 60$ & Cystoid macular oedema + vitritis \\
Ischaemic & $6 / 60$ & Cystoid macular oedema + optic atrophy \\
& PA & PL & Vitreous haemorrhage \\
& WJ & CF & Cystoid macular oedema \\
& KML & CF & Tractional retinal detachment \\
& MP & NPL & Branch retinal vein occlusion \\
& FL1 & Tractional retinal detachment \\
& FL2 & CyM & Vitreous hacular oedema \\
LS & CF & Optic atrophy + vitritis \\
\hline
\end{tabular}

$\mathrm{CF}$, counting fingers; HM, hand movements; PL, perception of light; NPL, no perception of light. 
Table III. The number of patients over the follow-up period who had either no systemic treatment, only prednisolone, or prednisolone with second-line immunosuppressants (cyclosporin A, azathioprine or colchicine)

\begin{tabular}{lcccc}
\hline Systemic treatment: & Nil & $\begin{array}{c}\text { Prednisolone } \\
\text { alone }\end{array}$ & $\begin{array}{c}\text { Prednisolone + second-line } \\
\text { immunosuppressants }\end{array}$ & Total \\
\hline Ischaemic RV & $1(5 \%)$ & $7(35 \%)$ & $12(60 \%)$ & $20(100 \%)$ \\
Non-ischaemic RV & $6(18 \%)$ & $17(52 \%)$ & $10(30 \%)$ & $33(100 \%)$ \\
$\begin{array}{l}p \text { value for ischaemic vs non-ischaemic RV } \\
\text { (Fisher's exact test) }\end{array}$ & 0.171 & 0.188 & 0.033 & \\
\hline
\end{tabular}

\section{Systemic Drugs}

Six of 33 of the non-ischaemic RV patients had no systemic treatment in the follow-up period, compared with only 1 patient in the ischaemic RV group. Over half of the non-ischaemic RV patients (17/33, $52 \%)$ were treated with systemic steroids alone compared with only 7 of $20(35 \%)$ of the ischaemic $\mathrm{RV}$ patients. A higher proportion of ischaemic RV patients had second-line immunosuppressants (cyclosporin A, azathioprine or colchicine) either singly or in combination during the follow-up period: 12 of $20(60 \%)$ compared with 10 of $33(30 \%)$ in the non-ischaemic RV group (Table III).

Twenty-two patients from both groups had second-line immunosuppressants during the follow-up period, of which $11(50 \%)$ had at least one eye with a final visual acuity of $6 / 60$ or less. Of these 11 patients, a higher proportion had ischaemic rather than nonischaemic disease (9/11 compared with 2/11: Fisher's exact test, $p=0.015)$.

\section{Ophthalmic Findings at Termination of the Study}

Visual Acuity and Causes of Grade IV Vision

The distribution of final visual acuity grades in both groups is as shown in Fig. 2. The patients with ischaemic RV fared worse than those with nonischaemic disease: 13 of 38 eyes in the ischaemic RV group had a final grade IV vision compared with only 4 of 63 in the non-ischaemic group (Fisher's exact text, $p=0.0005)$. The causes of grade IV vision are as outlined in Table IV. In the case of the nonischaemic RV patients, all 4 had uniocular poor vision and, for each patient, the eye in question had had poor vision at presentation. In the case of the ischaemic patients, of the 11 who had one or both eyes with a final vision of grade IV, 5 had an eye which had deteriorated from a higher visual grade during the follow-up period, and 5 had one or both eyes which had had grade IV acuity from the outset. One patient (FL1) had unilateral grade IV vision on entry but was blind in both eyes by the end of the study.

Of the 9 ischaemic patients who had a vitreous haemorrhage, 15 eyes were affected of which 13 $(86.7 \%)$ had a poor visual outcome (failure to maintain a visual acuity of $6 / 12$ or better, or to improve by at least one visual acuity grade). The causes of poor visual outcome were variable: 6 eyes
(46.2\%) had maculopathies, 2 (15.4\%) had tractional retinal detachments, and $2(15.4 \%)$ had recurrent vitreous haemorrhages. A further 2 eyes $(15.4 \%)$ had posterior capsular thickening following cataract surgery, and 1 eye $(7.6 \%)$ had optic atrophy secondary to retinal ischaemia. The poor visual outcome of these eyes occurred despite aggressive systemic and laser treatment. A significantly higher proportion of eyes with ischaemic $\mathrm{RV}$ fulfilled the criteria for a poor visual outcome compared with those with non-ischaemic RV (15/38 and 13/63 respectively; Fisher's exact test, $p=0.035)$. Only 1 patient (3\%) with non-ischaemic RV had a poor visual outcome in both eyes compared with 7 of 20 $(35 \%)$ in the ischaemic RV group (Fisher's exact text, $p=0.003$ ).
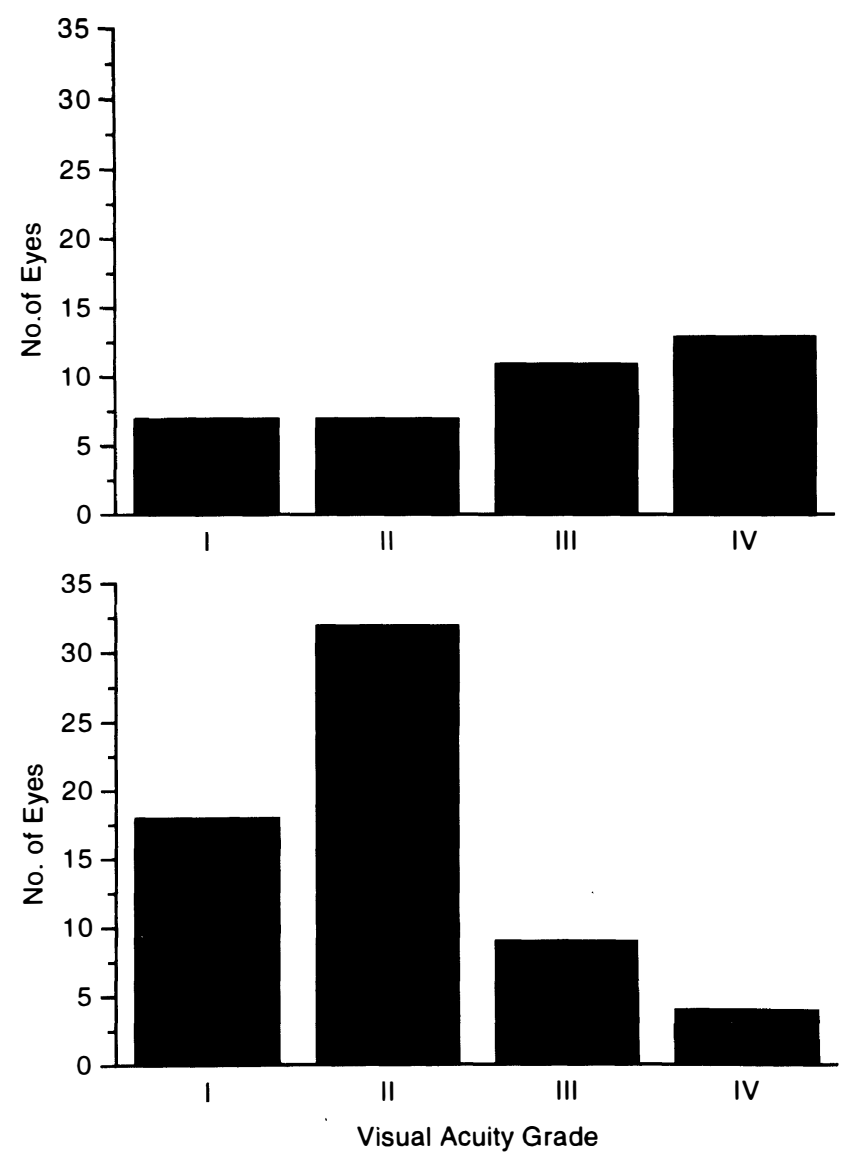

Fig. 2. The distribution of final visual acuity grades of ischaemic $R V$ (above) and non-ischaemic $R V$ patients (below). Visual acuity grades as for Fig. 1. 
Table IV. Causes of grade IV vision (6/60 or less) in RV patients at end of study period

\begin{tabular}{llll}
\hline & Patient & Visual acuity & Cause of poor vision \\
\hline Non-ischaemic & AI & $6 / 60$ & Macular epiretinal membrane \\
& HM & HM & Optic atrophy \\
& DP & $6 / 60$ & Maculopathy + cataract \\
Ischaemic & $6 / 60$ & Macular epiretinal membrane + cataract \\
& TA & PL & Optic atrophy \\
& SB & $6 / 60$ & Optic atrophy \\
AC & CF & Papillo-macular retinal infiltrates \\
& SC & PL & Posterior capsular thickening \\
WJ & EF & Vitreous haemorrhage \\
KM & NPL & Painfulopathy blind eye (tractional retinal detachment) \\
& MP & CF & Tractional retinal detachment \\
VS & $6 / 60$ & Cataract \\
FL1 & NPL & Maculopathy + posterior capsular thickening \\
& HM & Tractional retinal detachment \\
FP & HM & Vitreous haemorrhage \\
& & Optic atrophy + cataract \\
\hline
\end{tabular}

$\mathrm{CF}$, counting fingers; HM, hand movements; PL, perception of light; NPL, no perception of light.

\section{DISCUSSION}

This retrospective study has shown that patients with ischaemic RV have a significantly worse visual outcome than those with non-ischaemic RV. There was no difference in the age and sex distributions between the two groups, which is consistent with previous observations of RV patients. ${ }^{12}$

When we consider potential risk factors for poor visual outcome, smoking was more prevalent in the ischaemic $\mathrm{RV}$ group. This parallels our recent study of thrombophilic factors in RV, in which we found that a significantly higher proportion of ischaemic $\mathrm{RV}$ patients were smokers. ${ }^{13}$ In this smoking group, von Willebrand's factor (vWF) and fibrinogen levels were significantly higher than in their non-smoking counterparts. It is possible that both $\mathrm{vWF}$ and fibrinogen, which are raised in smokers, play a role in retinal capillary closure in $\mathrm{RV}$, although their increased levels can occur due to endothelial damage from smoking, and may therefore represent an epiphenomenon. $^{14,15}$ In this study, only 1 patient was diabetic (but had no diabetic retinopathy) and none had hypertension or a haemoglobinopathy, so the results were not complicated by concurrent vascular diseases.

Neither the duration of RV before referral nor the length of follow-up at St Thomas' was significantly different between the two groups. Therefore this cannot account either for the tendency of the ischaemic RV patients to have worse vision on entry into the study, or for the significantly worse visual outcome of the ischaemic group at the end. However, we are aware that a selection bias may exist since only complicated cases may have been referred to our unit.

This study has shown that the causes of poor vision in patients with RV are multifactorial, but that the main one is macular disease. In approximately onethird of eyes with grade IV vision at presentation, cystoid macular oedema (CMO) was a significant contributory factor. This resolved on systemic treatment in 4 of the 6 eyes, indicating that CMO, when adequately treated, is associated with a good prognosis. As would be expected, vitreous haemorrhage occurred in significantly more patients with ischaemic than non-ischaemic RV. This study has shown that eyes with ischaemic RV which have vitreous haemorrhages do not fare well despite aggressive treatment, and that causes of poor visual outcome in this group are also variable.

The rate of disease relapse was not significantly different between the two groups. This finding is consistent with the previous observation that the number of disease relapses does not vary between different $\mathrm{RV}$ types and that the relapse rate per se in $\mathrm{RV}$ does not appear to be an important factor in determining visual outcome. ${ }^{10}$

A significantly higher proportion of patients with ischaemic RV went on to second-line immunosuppressants, showing that these patients are more treatment resistant. This may be because the retinal ischaemia is made worse by the potentially procoagulant effects of immunosuppressants. ${ }^{16}$ This raises the question of whether anti-coagulant treatment should be given to patients with ischaemic RV who are on procoagulant immunosuppressive treatment.

Macular ischaemia had not been noted at the time of angiography in any of the RV patients. This may be because macular ischaemia, unassociated with predisposing systemic disease such as diabetes or local vaso-occlusive events, was not recognised until $1993 .{ }^{9}$ In addition, subtle irregularities of the foveal avascular zone were sometimes difficult to determine because of the degree of vitreous activity, leading to hazy fluorescein angiograms. In this study macular ischaemia was not a major cause of blindness but when present did contribute to poor visual outcome.

Overall, the visual outcome for the RV patients in this study was favourable; under one-third of all the patients had an eye with a final vision of grade IV. 
When grade IV vision did occur it was predominantly unilateral. None of the non-ischaemic RV patients had bilateral grade IV vision at either the beginning or the end of the follow-up period, and only $10 \%$ of the ischaemic RV patients had bilateral grade IV vision at the end of the study.

The causes of poor vision in $\mathrm{RV}$ are multifactorial, but macular disease is common and ischaemic RV patients with vitreous haemorrhage tend to fare badly. The worse visual outcome in ischaemic RV patients occurs despite aggressive systemic treatment with either prednisolone alone or in combination with second-line immunosuppressants, such as cyclosporin A. This raises the question as to whether other treatments with anti-coagulant properties should be considered for ischaemic RV.

This work was supported by the St Thomas' Endowments Committee and the Iris Fund for the Prevention of Blindness. The authors thank Mrs D. Paterson for typing the manuscript.

Key words: Retinal vasculitis, Visual outcome.

\section{REFERENCES}

1. Sanders MD. Retinal arteritis, retinal vasculitis and autoimmune retinal vasculitis. Eye 1987;1:441-65.

2. Brockhurst RJ, Schepens CL. Uveitis IV. Peripheral uveitis: the complication of retinal detachment. Arch Ophthalmol 1968;80:747-53.

3. Eales H. Cases of retinal haemorrhage associated with epistaxis and constipation. Birmingham Med Rev 1880;9:262.

4. Elliot AJ. Thirty year observation of patients with Eales disease. Am J Ophthalmol 1975;80:404-8.
5. Atmaca LS, Idil A, Gunduz K. Visualisation of retinal vasculitis in Eales disease. Ocul Immunol Inflamm 1993;1:41-8.

6. Charamis J. On the classification and management of the evolutionary course of Eales disease. Trans Ophthalmol Soc UK 1965;85:157-60.

7. Donders PC. Eales disease. Doc Ophthalmol 1958;12:1-21.

8. Malinowski SM, Pulido JS, Folk JC. Long term visual outcome and complications associated with pars planitis. Ophthalmology 1993;100:818-25.

9. Bentley CR, Stanford MR, Shilling JS, Sanders MD, Graham EM. Macular ischaemia in posterior uveitis. Eye 1993;7:411-4.

10. Stanford MR, Graham EM, Kasp E, Sanders MD, Dumonde DC. A longitudinal study of clinical and immunological findings in 52 patients with relapsing retinal vasculitis. Br J Ophthalmol 1988;72:442-7.

11. Howe LJ, Stanford MR, Edelsten C, Graham EM. The efficiency of systemic corticosteroids in sight-threatening retinal vasculitis. Eye 1994;8:443-7.

12. Graham EM, Stanford MR, Sanders MD, Kasp E, Dumonde DC. A point prevalence study of 150 patients with idiopathic retinal vasculitis. I. Diagnostic value of ophthalmological features. $\mathrm{Br} \mathrm{J}$ Ophthalmol 1986;73:714-21.

13. Palmer HE, Jurd KM, Hunt BJ, Zaman AG, Stanford MR, Sanders MD, Graham EM. Thrombophilic factors in ischaemic and non-ischaemic retinal vasculitis. Eye 1995;9:507-12.

14. Blann AD, McCollum CN. Adverse influence of cigarette smoking on the endothelium. Thromb Haemost 1993;70:707-11.

15. Ernst E. Fibrinogen: an independent risk factor for cardiovascular disease. BMJ 1991;303:596-7.

16. Vanrenterghem Y, Roels L, Lerut T, Gruwez J, et al. Thromboembolic complications and haemostatic changes in cyclosporin-treated cadaveric kidney allograft recipients. Lancet 1985;8436:999-1002. 